\title{
Energy Minimization in Wireless Sensor Networks Using Multi Hop Transmission
}

\author{
${ }^{1}$ Radarapu bharathi, ${ }^{2}$ Tamatam Neeraja \\ ${ }^{1,2}$ Department of computer Science and Information Technology Kamala Institute Of Technology and \\ Science,Singapur,Huzurabad.
}

\begin{abstract}
Wireless technology has enormous potential to change the way people and things communicate. Wireless cellular networks allow people on the move to communicate with anyone or anything using a range of multimedia services. Of all potential wireless applications, sensor networks are special due to their emphasis on communication between devices. In addition, these networks have hard energy constraints since each node is powered by a small battery that may not be rechargeable or renewable. Therefore, reducing energy consumption is the most important design consideration for such networks. A sensor network is composed of dozens, or even thousands of nodes, connected in a systematic way, as shown in Fig. 1.1. This figure indicates that the information from the sensors flows into a hub node. As shown in the figure, if the source node is far away from the hub node, intermediate nodes can help with relaying via multihop transmissions. These networked sensors are distributed to collect information on entities of interest.

A wireless sensor network (WSN) consists of a group of self-organizing, lightweight sensor nodes that are used to cooperatively monitor physical or environmental conditions. Commonly monitored parameters include temperature, sound, humidity, vibration, pressure and motion. Each sensor node in a WSN is equipped with a radio transmitter, several sensors, a battery unit and a microcontroller. Although the WSN research was initially motivated by military applications, wireless sensor networks are now used in many industrial and public service areas. Sensor nodes are inexpensive portable devices with limited processing power and energy resources. The Sensor nodes can be used to collect information from the environment, locally process this data and transmit the sensed data back to the user. Sensor nodes consist of five main components : a computing unit, a communication unit, a sensing unit, a memory unit, and a power supply unit. The computing unit consists of a microprocessor. The microprocessor is responsible for managing the communication protocols, processing collected data from the on-board sensors, and performing the power management. Each sensor node has a single communication unit that is able to transmit and receive packets. This unit combines the functionality of both transmitter and receiver

Although all sensing, computing and communication operations consume energy, data communication requires more energy than sensing and computing. Thus, reducing data communication between sensor nodes can improve the energy efficiency and extend the lifetime of sensor networks. A WSN is able to self-configure its operation and manage its connectivity. A WSN is also able to tolerate malfunctioning nodes and integrate new nodes in the network since node failure is common in WSN applications. Because of the limited power and transmission range in a large sensor network, the communication between sensor nodes must be multihop. Data from a source sensor node relayed by a number of intermediate nodes before it reaches the final destination. The Collaboration between sensor nodes and in-network processing are necessary in sensor networks since a single node may not have all the data concerning some event of interest .
\end{abstract}

\section{Challenges And Motivations}

In previous works, energy efficiency of cooperative transmissions over a single hop was investigated and compared to the traditional SISO transmissions. The capacity of a large Gaussian relay network, where a source cooperates with relay nodes to transmit to a sink node is investigated. In our work, we investigate energy efficient routing in multi-hop wireless networks with cooperative transmissions when the channel is slowlyvarying. The key advantage provided by the cooperative transmissions considered in this work is the increase in the transmission range due to diversity gain when all radios transmit at the same fixed power level as in traditional SISO systems.

The reliability issue in the data transport protocol usually involves loss recovery, congestion control, or both. Most of the reliable data transport protocols either use a retransmission-based loss recovery approach or a redundant data transmission method (sending multiple copies of a data packet into the network). As in many other types of networks, congestion in wireless sensor networks can have a significant impact on quality of service. 
After studying the design challenges of data transport protocols and existing reliable data transport protocols in wireless sensor networks, it was found that no existing protocol has all of the following characteristics:

- Full reliability ( $100 \%$ reliable data delivery) is provided unless there are unavoidable packet drops due to buffer overflow.

- Recovery from packet loss can be achieved with low system overhead and reduced communication cost and delay compared to conventional protocols.

- Lost packets can be recovered as quickly as possible, while at the same time not interfering with normal data transmission.

- A specified level of robustness can be provided.

- The protocol is robust to node failure and route changes.

- Fresh data has higher priority in the network and is able to be sent as soon as possible.

- The protocol is scalable and easy to implement.

Sensor networks are desired to be reliable, expandable, and easily implemental. They should also be able to collect information with low delay and have a long lifetime.However, before we can achieve these goals, we must be able to address the research problems arising from the following three areas: we need to design sensitive and low cost sensors to collect information; we need to develop efficient networking protocols to transmit information from the source nodes to the fusion center; and we need to explore optimal algorithms to process and abstract the core intelligence from the raw data collected from all nodes. .

We assume that each information bit collected by a sensor is useful for a finite amount of time; after this time the information may become irrelevant. Hence, all the bits collected by the sensors need to be communicated to a hub node before a certain deadline. Therefore, the maximum end-to-end transmission delay for each bit must be controlled to meet a given deadline under the hard energy constraint. Since all layers of the protocol stack affect the energy consumption and delay for the end-to-end transmission of each bit, an efficient system requires a joint design across all these layers that incorporate the underlying hardware characteristics.

The main functions of each individual layer are described as follows:

1. The hardware layer is composed of the fundamental hardware blocks where the upper layer algorithms are implemented. Since all the power is consumed physically in this layer, it is must be considered in a cross-layer energy minimization framework.

2. The link layer, also referred to as the physical layer, deals primarily with transmitting bits reliably over a point-to-point wireless link. The design tradeoffs associated with the link layer include modulation, coding, diversity, adaptive techniques, MIMO, equalization, multi-carrier modulation, and spread spectrum.

3. The MAC layer controls how different users share the given spectrum and ensures reliable packet transmissions. Allocation of signaling dimensions to different users is done through either deterministic access or random access. For deterministic access, the signaling dimensions are divided into dedicated channels, where the most common methods are Time Division Multiple Access (TDMA), Frequency Division Multiple Access (FDMA), and Code Division Multiple Access (CDMA). For random access, the channels are assigned to active users dynamically, and the most common methods are different forms of ALOHA, Carrier Sensing Multiple Access (CSMA), and scheduling.

4. The network layer establishes and maintains end-to-end connections in the network. The main functions of the network layer in a sensor network are neighbor discovery, routing, and dynamic resource allocation.

5. The transport layer provides the end-to-end functions of retransmission, error recovery, reordering, and flow control. The most common protocol used at this layer is the Transport Protocol (TCP).

\section{Problem Outline}

In wireless networks, energy efficiency is a dominating design criterion. It is well-known that for the same throughput requirement multi input multi output (MIMO) systems require less transmission energy than single input single output (SISO) systems in the presence of fading . However, it is usually infeasible to mount multiple antennas on small wireless devices due to the required minimum separation of these antennas. To achieve MIMO gains in wireless networks, cooperative (virtual) MIMO techniques have been proposed. There is an increasing interest in translating the advantages of using virtual MIMO at the physical layer into higher layer performance benefits to maximize network throughput, or minimize total energy consumption and end-toend delay. In previous works, energy efficiency of cooperative transmissions over a single hop was investigated and compared to the traditional SISO transmissions. The capacity of a large gaussian relay network, where a source cooperates with relay nodes to transmit to a sink node is investigated in . In our work, we investigate energy efficient routing in multi-hop wireless networks with cooperative transmissions when the channel is slowly-varying. 


\section{Objective}

The key advantage provided by the cooperative transmissions considered in this work is the increase in the transmission range due to diversity gain when all radio transmit at the same fixed power level as in traditional SISO systems. Our objective is to determine in a multi-hop network, the optimal number of cooperating nodes per hop to minimize the end to- end total energy consumption while satisfying an outage probability requirement at each hop. In order to identify the effect of the number of cooperating nodes on energy consumption, all other parameters, i.e., transmission power and reliability are kept constant. The theoretical analysis of this problem is performed for networks with unlimited node density. Our results indicate that cooperative transmission is especially useful in multi-hop networks with low propagation loss coefficient, stricter outage probability requirement, and lower transmission power level. A new greedy geographical routing algorithm suitable for vMISO transmissions is designed to demonstrate the applicability of our results for more general networks.

\section{MIMO TRANSMISSION MODEL}

We focus on a single-user communication model and consider a point-to-point link where the transmitter is equipped with $n T$ antennas and the receiver employs $n R$ antennas (see Figure 1.4). Next to the single user assumption in the depiction as point-to-point link, we suppose that no intersymbol interference (ISI) occurs. This implies that the bandwidth of the transmitted signal is very small and can be assumed frequency- ${ }^{\circ}$ at (narrowband assumption), so that each signal path can be represented by a complex-valued gain factor. For practical purposes, it is common to model the channel as frequency- ${ }^{\circ}$ at whenever the bandwidth of the system is smaller than the inverse of the delay spread of the channel.

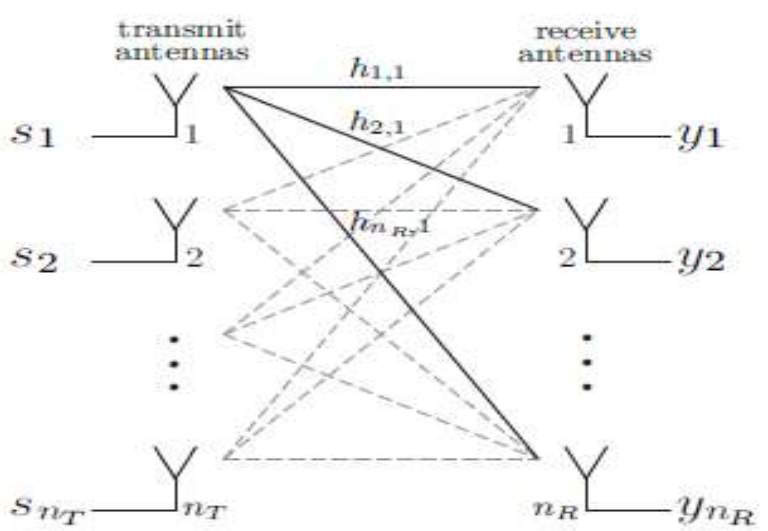

A MIMO channel with $n T$ transmit and $n R$ receive antennas

If the channel is frequency selective, one could use an OFDM (orthogonal frequency-division multiplexing) system, to turn the MIMO channel into a set of parallel frequency- ${ }^{\circ}$ at MIMO channels, of which each obeys our stated assumptions. In addition to these restrictions, we will further assume, that we are operating in a time- invariant setup. These assumptions allow us to use the standard complex-valued baseband representation of narrowband signals that can be written in a discrete form (omitting the dependency on time). Now let $h i ; j$ be the complex-valued path gain from transmit antenna $j$ to receive antenna $i$ (the fading coe \pm cient). If at a certain time instant the complex-valued signals $f s 1 ;: \therefore ; s n T g$ are transmitted via the $n T$ antennas, respectively, the received signal at antenna $i$ can be expressed as

$$
y_{i}=\sum_{j=1}^{n_{T}} h_{i, j} s_{j}+n_{i},
$$

where $n i$ represents additive noise, which will be treated later in this chapter. This linear relation can be easily written in a matrix framework. Thus, let s be a vector of size $n T$ containing the transmitted values, and y be a vector of size $n R$ containing the received values, respectively. Certainly, we have s $\in \mathrm{C} n T$ and y $\in \mathrm{C} n R$. Moreover, if we define the channel transfer matrix $\mathrm{H}$ as

$$
\mathbf{H}=\left[\begin{array}{cccc}
h_{1,1} & h_{1,2} & \ldots & h_{1, n_{T}} \\
h_{2,1} & h_{2,2} & \ldots & h_{2, n_{T}} \\
\vdots & \vdots & \ddots & \vdots \\
h_{n_{R}, 1} & h_{n_{R}, 2} & \ldots & h_{n_{R}, n_{T}}
\end{array}\right],
$$


we obtain

$$
\mathbf{y}=\mathbf{H s}+\mathbf{n} . \quad \text { eq(1.3) }
$$

This is the same matrix notation as it is used in the majority of the publications in this field, e.g. . This relation, denoting a transmission only over one symbol interval, is easily adapted to the case that several consecutive vectors $f \mathrm{~s} 1$; s2; : : ; s $L g$ are transmitted (here, $L$ denotes the total number of symbol intervals used for transmission) over the channel. Therefore, we arrange the transmitted, the received and the noise vectors in the matrices

$$
\mathbf{S}=\left[\mathbf{s}_{1}, \mathbf{s}_{2}, \cdots, \mathbf{s}_{L}\right], \quad \mathbf{Y}=\left[\mathbf{y}_{1}, \mathbf{y}_{2}, \cdots, \mathbf{y}_{L}\right], \quad \mathbf{N}=\left[\mathbf{n}_{1}, \mathbf{n}_{2}, \cdots, \mathbf{n}_{L}\right],
$$

eq(1.4)

respectively. The associated block transmission model is

$$
\left[\begin{array}{ccc}
y_{1,1} & \cdots & y_{1, L} \\
y_{2,1} & \cdots & y_{2, L} \\
\vdots & \ddots & \vdots \\
y_{n_{R}, 1} & \cdots & y_{n_{R}, L}
\end{array}\right]=\left[\begin{array}{ccc}
h_{1,1} & \ldots & h_{1, n_{T}} \\
h_{2,1} & \cdots & h_{2, n_{T}} \\
\vdots & \ddots & \vdots \\
h_{n_{R}, 1} & \cdots & h_{n_{R}, n_{T}}
\end{array}\right]\left[\begin{array}{ccc}
s_{1,1} & \ldots & s_{1, L} \\
s_{2,1} & \cdots & s_{2, L} \\
\vdots & \ddots & \vdots \\
s_{n_{T}, 1} & \cdots & s_{n_{T}, L}
\end{array}\right]+\left[\begin{array}{ccc}
n_{1,1} & \cdots & n_{1, L} \\
n_{2,1} & \cdots & n_{2, L} \\
\vdots & \ddots & \vdots \\
n_{n_{R}, 1} & \cdots & n_{n_{R}, L}
\end{array}\right]
$$

eq(1.5)

or equivalently

$$
\mathbf{Y}=\mathbf{H S}+\mathbf{N} \text {. }
$$

$\operatorname{eq}(1.6)$

Fading The elements of the matrix $\mathrm{H}$ correspond to the complex-valued channel gains between each transmit and receive antenna. For the purpose of assessing and predicting the performance of a communication system, it is necessary to postulate a statistical distribution of these elements . This is also true to some degree for the design of well performing receivers, in the sense that knowledge of the statistical behavior of $\mathrm{H}$ could potentially be used to improve the performance of receivers. Throughout this thesis, we will assume that the elements of the channel matrix $\mathrm{H}$ are zero- mean complex-valued Gaussian random variables with unit variance. This assumption is made to model the fading effects induced by local scattering in the absence of line-of-sight components. Consequently, the magnitudes of the channel gains hi;j have a Rayleigh distribution, or equivalently, $j h i ; j j 2$ are exponentially distributed $[8,14]$. The presence of line-of-sight components can be modeled by letting $h i ; j$ have a Gaussian distribution with a non-zero mean (this is also called Ricean fading). After having identified the possibilities to model the complex-valued channel path gains, it remains to check a possible correlation between these entries. In this work, we make a commonly made assumption on $\mathrm{H}$, i.e. that the elements of $\mathrm{H}$ are statistically independent. Although this assumption again tends to yield mathematical expressions that are easy to deal with, and allows the identification of fundamental performance limits, it is usually a rough approximation. In practice, the complex path gains fhi;jg are correlated by an amount that depends on the propagation environment as well as the polarization of the antenna elements and the spacing between them.

\section{DESIGN APPROACH OF VMISO SYSTEMS}

The channel is modeled as a Rayleigh flat-fading channel, where each node transmits with a fixed power level, $P 0$. The receiver has the full channel state information (CSI), but the transmitters do not estimate the channel. Let $N 0$ be the one-sided noise spectral density and $\alpha 0$ be the complex Gaussian distributed random variable, $N c(0,1)$, characterizing the Rayleigh flat fading channel. The instantaneous signalto- noise-ratio (SNR) at a SISO receiver is, $S N R^{S I S O}=\frac{P_{0}}{N_{0}}\left|\alpha_{0}\right|^{2} d_{0}^{-\beta}$, where $\beta$ is the path loss coefficient and $d 0$ is the transmission range. In vMISO systems, a set of cooperating nodes emulate the antenna array of real MISO systems. vMISO systems can provide diversity gain over SISO systems due to the transmission of data over multiple independent channels. We consider decode and forward cooperation scheme, where initially, the head node transmits the original data to the relay nodes .

The cooperative transmission begins once all relay nodes receive, and correctly decode the original data. In order to leverage the benefits of space-diversity, data is encoded by a space time block code (STBC) with code rate $r n=k / k n \leq 1$. The head node and $n-1$ relay nodes simultaneously transmit over $k n$ time slots in order to transfer $k$ information bits. Let $\alpha i$ and $d i$ be the fading coefficient of the channel, and the distance between the $i$ th cooperating node and the destination The received SNR in vMISO systems with $n$ cooperating nodes is , $S N R v M I S O=\frac{P_{0}}{N_{0}} \sum_{i=0}^{n-1}\left|\alpha_{i}\right|^{2} d_{i}^{-\beta}$. Depending on the relative locations of the relay nodes and the head node, the symbols may arrive at the receiver with different received powers and relative delays. A numerical analysis on whether this difference can cause significant performance degradation was provided in. 
In, the cumulative distribution function for relative delay and power difference is numerically determined for line-of-sight propagation model and assuming that the relay nodes are uniformly distributed within the SISO range (taken as $250 \mathrm{~m}$ ). The results indicate that when the next hop node is further away than the SISO range, then the delay difference between the signals from any two relay nodes is at most $0.6 \mu$ s for more than $80 \%$ of the time, and the power difference is less than $5 \mathrm{~dB}$ for more than $85 \%$ of the time. The asynchronous reception at the receiver can cause inter-symbol interference (ISI), but this can be overcome with such methods as timereverse space-time codes, or space-time OFDM. Therefore, in almost all cases wherein cooperative transmissions are used, the diversity gain is only dependent on the number of cooperating transmitters, and not on the physical location of these transmitters. Spatial diversity can help transmit to larger distances, while satisfying the same bit error rate (BER) requirement with the same transmit power. However, the analysis involving BER involves complicated mathematical functions. A more general and tractable way to capture the link quality is by outage probability, $p$, defined as the probability that the received Signal-to-Noise-Ratio (SNR) falls below a certain threshold, SNRth. SNR threshold is a widely used metric previously employed for transmit power control in cell phones. We assume that symbols are transmitted at the same transmission rate with SISO and vMISO systems, and the receiver performs linear combination of the received symbols. In this setting, it is natural to assume that SNRth is same for both SISO and vMISO systems. Therefore, the outage probability with vMISO and SISO are given as,

$$
\begin{aligned}
p & =\operatorname{Pr}\left[\frac{P_{0}}{N_{0}} \sum_{i=0}^{n-1}\left|\alpha_{i}\right|^{2} d_{i}^{-\beta} \leq S N R_{t h}\right] \\
& =\operatorname{Pr}\left[\frac{P_{0}}{N_{0}}\left|\alpha_{0}\right|^{2} d_{0}^{-\beta} \leq S N R_{t h}\right] .
\end{aligned}
$$

\section{Single-Hop vmiso vs. Multi-Hop SISO}

We first compare the energy efficiency of direct vMISO transmission and multi-hop SISO transmission. Let $E v M I S O(k, d v, n)$ be the total energy cost of transmitting $k$ bits of information to a distance of $d v$ in a single vMISO transmission with $n$ cooperating nodes. Initially, the head node broadcasts $k$ bits of original data to its relay nodes consuming $k\left(E_{e}^{t}+E_{a}\right)$ units of energy in the process. Also, $n-1$ relay nodes consume $k(n-1) E r$ e units of energy during reception. We assume that all nodes are pre-loaded with a table of space-time block code matrices, such as those given in for different levels of transmit diversity. Each column of the matrix corresponds to a block duration (time), whereas, each row holds the symbols to be transmitted by each relay node. Next, each cooperating node transmits blocks of in total of $k n$ bits when code rate is $r n=k / k n$, collectively consuming $n k n(E t e+E a)$ units of total energy. The destination combines received bits linearly, and consumes approximately $k n E_{e}{ }^{r}$ units of energy.Therefore, when

$$
\begin{aligned}
& E_{e}^{t}=E_{e}^{r}=E_{e}, \\
& E_{v M I S O}\left(k, d_{v}, n\right)=k E_{e}\left[n+\frac{E_{a}}{E_{e}}+\frac{1}{r_{n}}\left(n\left(1+\frac{E_{a}}{E_{e}}\right)+1\right)\right],{ }_{\text {for }} n \geq 2 .
\end{aligned}
$$

Similarly,a SISO transmission consumes $\operatorname{ESISO}(k, d 0)=k E_{e}^{t}+k E_{a}+k E_{e}^{r}$ units of energy while transmitting $k$ bits to a distance $d 0(p)$, where $d 0(p)=d_{\text {nom }}\left(\frac{\gamma_{0}(p)}{\gamma_{0}\left(p_{\text {nom }}\right)}\right)^{1 / \beta}$.

\section{MULTI-HOP VMISO VS. MULTI-HOP SISO}

Now, our objective is to find the optimal transmission strategy at each hop of a multi-hop vMISO system that minimizes the total energy consumption. We first determine the optimal number of cooperating nodes, nopt, at each hop for a given outage probability requirement, $p$. Note that under a high node density regime, nopt is the same at each hop by symmetry. 1) Optimal transmission strategy given the required link outage probability: Let $\operatorname{Emhop}(k, D, n)$ be the total energy consumption of transmitting $k$ bits to a distance of $D$ meters with a multi-hop SISO or a multi-hop vMISO system with $n$ cooperating nodes, and $M=\left\lceil\frac{D}{d_{0}(p)}\right\rceil$ and $K=\left\lceil\frac{D}{d_{v}(n, p)}\right\rceil$ be the number of hops needed to transmit a symbol to a distance of $D$ with multi-hop SISO and vMISO systems, respectively. Then, the optimal number of cooperating nodes is determined by solving the following optimization problem: 


$$
\begin{aligned}
\min _{n} E_{\text {mhop }}(k, D, n)= & \min \left\{M E_{S I S O}\left(k, d_{0}(p)\right),\right. \\
& \left.\min _{n \in \mathbb{Z}^{+}: n \geq 2}\left[K E_{v M I S O}\left(k, d_{v}(n, p), n\right)\right]\right\}
\end{aligned}
$$

Note that the solution of (3) may give SISO as the optimal solution. (3) is a nonlinear integer program, since $n, K$ and $M$ are nonnegative integers. The effective solution space of (3) is a narrow domain in integer space, since STBC is not efficient for large $n$. Therefore, we determine nopt by enumeration. We calculated nopt numerically for varying $p$, where we used $r n$ values given in. It is observed that cooperation is especially preferred when $p$ is low, since the number of hops taken by vMISO is much lower than SISO. This reduction in number of hops compensates the higher per hop energy consumption of vMISO. For high outage probability, nopt decreases, and converges to $n=1$, when $p \approx 0.06$, because for $p>0.05, \operatorname{Iv}(n, p)$ remains approximately constant. Thus, for $p>0.05$, and $\beta=2$, SISO is preferred. Also, nopt is lower for higher $\beta$, since the transmission energy increases with $\beta$. 2) Optimal transmission strategy and end-to-end reliability: Now, we also consider the end-to-end reliability of the transmissions. If a transmission fails on a link, it is retransmitted. Link failure is presumed to be independent and unpredictable, so our objective is to minimize the total average energy cost by determining nopt at every hop. Assuming that the channel is slowly varying, a vMISO transmission fails with outage probability, $p$. The number of transmissions until the first success is a geometric random variable, and the expected number of cooperative transmissions is calculated from $1 /(1-p)$. The cooperative transmission only begins if all of the relay nodes correctly decode the original symbol. The probability that none of the relay nodes can decode the symbol correctly is $1-(1-p) n-1$. Thus, the average number of broadcasts at the first phase of vMISO transmissions is calculated as $1 /(1-p) n-1$. Then, the total average energy cost of multi-hop vMISO is

$$
\begin{aligned}
\mathbf{E}\left[E_{v M I S O}(k, D, n, p)\right]=K E_{e} k & {\left[\frac{n+\frac{E_{a}}{E_{e}}}{(1-p)^{n-1}}\right.} \\
& \left.+\frac{n\left(1+\frac{E_{a}}{E_{c}}\right)+1}{(1-p) r_{n}}\right]
\end{aligned}
$$

Similarly, the total average energy cost of multi-hop SISO is

$$
\mathrm{E}\left[E_{S I S O}(k, D, p)\right]=M E_{e} k \frac{2+\frac{E_{a}}{E_{c}}}{1-p} \text {. }
$$

\section{Design Methodology}

We considered decode and forward cooperation scheme, where initially, the head node transmits the original data to the relay nodes. The cooperative transmission begins once all relay nodes receive, and correctly decode the original data.

\section{Single-Hop vMISO vs. Multi-Hop SISO}

- We first compare the energy efficiency of direct vMISO transmission and multi-hop SISO transmission.

- Let $E_{\mathrm{v}} M I S O\left(k, d_{v}, n\right)$ be the total energy cost of transmitting $k$ bits of information to a distance of $d v$ in a single vMISO transmission with $n$ cooperating nodes.

- Initially, the head node broadcasts $k$ bits of original data to its relay nodes consuming $k\left(E_{e}^{t}+E_{a}\right)$ units of energy in the process. Also, $n-1$ relay nodes consume $k(n-1) E_{e}^{r}$ units of energy during reception.

- Next, each cooperating node transmits total of $k_{n}$ bits when code rate is $r_{n}=k / k_{n}$, collectively consuming $n k_{n}$ $\left(E^{t} e+E a\right)$ units of total energy. The destination combines received bits linearly, and consumes approximately $k_{n} E^{r} e$ units of energy. When $\mathrm{E}_{\mathrm{e}}^{\mathrm{t}}=\mathrm{E}_{\mathrm{e}}^{\mathrm{r}}=\mathrm{E}_{\mathrm{e}}, E v M I S O(k, d v, n)=\mathrm{kE}_{\mathrm{e}}\left[\mathrm{n}+\mathrm{E}_{\mathrm{a}} / \mathrm{E}_{\mathrm{e}}+1 / \mathrm{r}_{\mathrm{n}}(\mathrm{n}(1\right.$ $\left.\left.\left.+\mathrm{E}_{\mathrm{a}} / \mathrm{E}_{\mathrm{e}}\right)+1\right)\right]$

\section{Multi-Hop vMISO vs. Multi-Hop SISO}

- Find the optimal transmission strategy at each hop of a multi-hop vMISO system that minimizes the total energy consumption.

- We first determine the optimal number of cooperating nodes, $n_{\text {opt }}$, at each hop for a given outage probability requirement, $p$. 
- Let $\mathrm{E}_{m \text {-hop }}(k, D, n)$ be the total energy consumption of transmitting $k$ bits to a distance of $D$ meters with a multi-hop SISO or a multi-hop vMISO system with $n$ cooperating nodes(range), and $M=\left[D / d_{0}(p)\right]$ and $K$ $=\left[D / d_{v}(n, p)\right]$ be the number of hops needed to transmit a symbol to a distance of $D$ with multi-hop SISO and vMISO systems, respectively.

- Then, the optimal number of cooperating nodes is determined by solving the following optimization problem: $\min _{n} \mathrm{E}_{m-h o p}(k, D, n)=\min \left\{\mathrm{E}_{\mathrm{SISO}}\left(k, d_{0}(p)\right), \min _{n}\left[E_{v M I S O}\left(k, d_{v}(n, p)\right.\right.\right.$

\section{FLOWCHART}

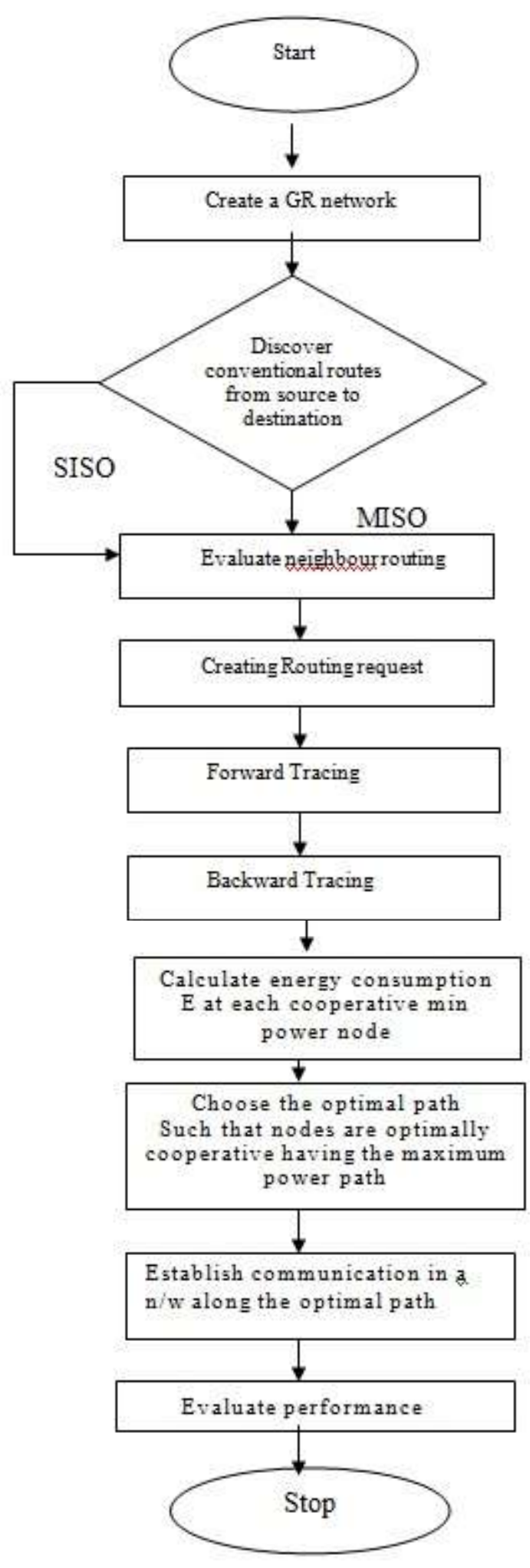




\section{Conclusion:}

We studied the energy-efficiency of a general multi-hop vMISO system. Under high node density regime, we determined the optimal cardinality of cooperation sets according to the required outage probability, transmission power and path loss coefficient. We demonstrated that our results can be used to design practical vMISO routing algorithms

suitable for more general networks. We performed simulation studies with the proposed vMISO GR algorithm. We considered a $30 \mathrm{~m} 730 \mathrm{~m}$ square area, where the nodes are randomly distributed, and $s$ and $t$ lie at the opposite corners of this area. We performed the simulations for varying node densities, and our results represent the average of the measurements over 20 random topologies for each node density. The transmissions are attenuated by a random Rayleigh distributed amount, and if a transmission is unsuccessful, i.e., received SNR is less than $S N R t h$, it is repeated. We used the values $S N R t h=14 \mathrm{~dB}, N 0=-40 \mathrm{dBm}$, dnom $=10 \mathrm{~m}$, pnom $=$ $0.1, p=5 \bar{\nu} 10-3$, and $\beta=3$ in the simulations.

\section{Reference}

[1] J. N. Laneman, "Cooperative diversity in wireless networks: algorithms and architectures," Ph.D. thesis, Massachusetts Institute of Technology, Cambridge, MA, Aug. 2002.

[2] X. Li, M. Chen, and W. Liu, "Application of STBC-encoded cooperative transmissions in wireless sensor networks," IEEE Signal Processing Lett., vol. 12, no. 2, pp. 134-137, Feb. 2005.

[3] S. Cui, A. J. Goldsmith, and A. Bahai, "Energy-efficiency of MIMO and cooperative MIMO in sensor networks," IEEE J. Select. Areas Commun., vol. 22, no. 6, pp. 1089-1098, Aug. 2004.

[4] S. Cui, A. J. Goldsmith, and A. Bahai, "Cross-layer design of energy constrained networks using cooperative MIMO techniques," EURASIP J. Applied Signal Processing, vol. 86, no. 8, pp. 1804-1814, Aug. 2006.

[5] G. Jakllari, S. V. Krishnamurthy, M. Faloutsos, P. V. Krishnamurthy, and O. Ercetin, "A cross-layer framework for exploiting virtual MISO links in mobile ad hoc networks,” IEEE Trans. Mobile Computing, vol. 6, no. 6, pp. 579-594, June 2007.

[6] S. K. Jayaweera, "Virtual MIMO-based cooperative communications architecture for energy-constrained wireless sensor networks," IEEE Trans. Wireless Commun., vol. 5, no. 5, pp. 984-989, May 2006.

[7] V. Tarokh, H. Jafarkhani, and A. R. Calderbank, "Space-time block codes from orthogonal designs," IEEE Trans. Inform. Theory, vol. 45, no. 5, pp. 1456-1467, July 1999. 